\title{
45. PERTURBATIONS OF THE LINE OF NODES OF THE LEONIDS DURING THE YEARS 1866-1966
}

\author{
V. GuTH \\ (Astronomical Institute of the Czechoslovak Academy of Sciences, Ondrejov)
}

The aim of our investigation was the determination of the epoch of the Leonid maximum for the return in 1966 . We applied the same method, which has been used by Stoney and Downing (1899) for the investigation of motion of Leonids in 1899, e.g. method of variation of elements. Our attention has been concentrated on the motion of the position of the line of nodes, which is one of the important characteristics for the epoch of maximum activity of the shower. We started with the Adams elements deduced from the splendid appearance of Leonids 1866, as did Stoney and Downing. We chose a 40-day interval for the integration step of perturbations as the most convenient, because all the positions of the great planets are tabulated with this interval in Washington tables (Eckert et al., 1951). Only the perturbations due to Jupiter, Saturn and Uranus during the years 1866-1966 have been considered as decisive for the motion of the Leonids' nodes. The computations for the epoch 1866-99-divided into five parts - have been compared with those obtained by Stoney and Downing, with the results shown in Table 1.

\section{Table 1}

\section{Perturbations of the ascending node}

Interval
$\begin{array}{lr}1866 & \text { XI. } 13 \\ 1871 & \text { V. } 3 \\ 1894 & \text { XII. } 28 \\ 1897 & \text { XII. } 30 \\ 1899 & \text { V. } 18 \\ 1900 & \text { I. } 27\end{array}$

Minsk-22

Stoney and
Downing

M-SD
$+25: 92$
$+7 ! .02$
$-1: 92$
$+25: 44$
$+1: 02$
$+29 ! 33$
$+7: 15$
$-1: 69$
$+70 ! 83$
$+0 \div 09$

$-3: 41$

$-0: 13$

$-0.23$

$+45: 39$ !

$+0 ! 93$

The results show a fair agreement for the first three intervals, but they disagree for the fourth one. As a cause of this disagreement we consider the possibility that Stoney and Downing left the elements unchanged for too long a time regardless of the large perturbations, especially in the major axis. 
Our primary result is the position of the nodes, but instead of this we can give immediately the time of passage of the Earth through the Leonid orbit plane. This is given in Table 2 .

The deduced value for the year 1966 corresponds to November 17.48 UT (epoch $1966)$ and is in very good agreement with observations of that year (17.50 UT).

\section{Table 2}

\section{Passage of the Earth through the Leonid orbit plane}

$\begin{array}{lcc}\text { Date (epoch 1950) } & \Omega=\odot \lambda(1950) & r-R \\ & & \\ 1866 \text { XI. } 15.55 & 232.596 & 0.000 \\ 1899 \text { XI. } 16.60 & 233.646 & -0.013 \\ 1933 \text { XI. } 17.02 & 234.113 & -0.019 \\ 1966 \text { XI. } 17.38 & 234.430 & -0.020 \\ 1967 \text { XI. } 17.36 & 234.410 & -\end{array}$

The prediction for the year 1967 (epoch 1967) 17.72 UT promises better conditions for observers in Asia and Europe than in the year 1966; however, it is 0.28 day earlier than the prediction of Kazimirčak-Polonskaja et al. (1967).

To consider the influence of perturbations on the different parts of the meteor stream the passage through perihelion was changed by \pm 360 and \pm 720 days, all other elements having been unchanged. Further, the perturbations of the line of nodes in connection with the period of revolution of the stream were studied, changing the original period of 33.25 years by \pm 0.75 years. The results of our calculations are shown in Figure 1.

Also some observations of maxima of Leonid activity according to their richness in different years are marked. Comparing the scatter of observed maxima with the scatter of the perturbation curves we see that the gravitational perturbation effect is sufficient to explain the variety of observations. Our results obviously did not reach the detailed analysis and accuracy of the Soviet astronomers (Kazimirčak-Polonskaja et al., 1967), which appeared in the meantime and about which we have just heard, but they show at least the importance of the perturbation calculations.

The gravitional field of Earth obviously influences the orbits of meteors passing nearby the Earth. The greatest positive change of the major axis takes place 'on the top' with an amount $a+3.06 \mathrm{AU}$ and a negative one 'at the bottom' of the Earth's sphere: $a-2.06 \mathrm{AU}$. The situation is shown in Figure 2, which represents the view of our Earth from the direction of the apparent radiant for the time of maximum activity in 1966, November 17, 11:55 UT. It clearly points out the observational conditions for different places on the Earth at that time.

To obtain more reliable hourly rates for the meteor showers of extremely high activity it seems to be suitable to use recording by photography with equal exposures 


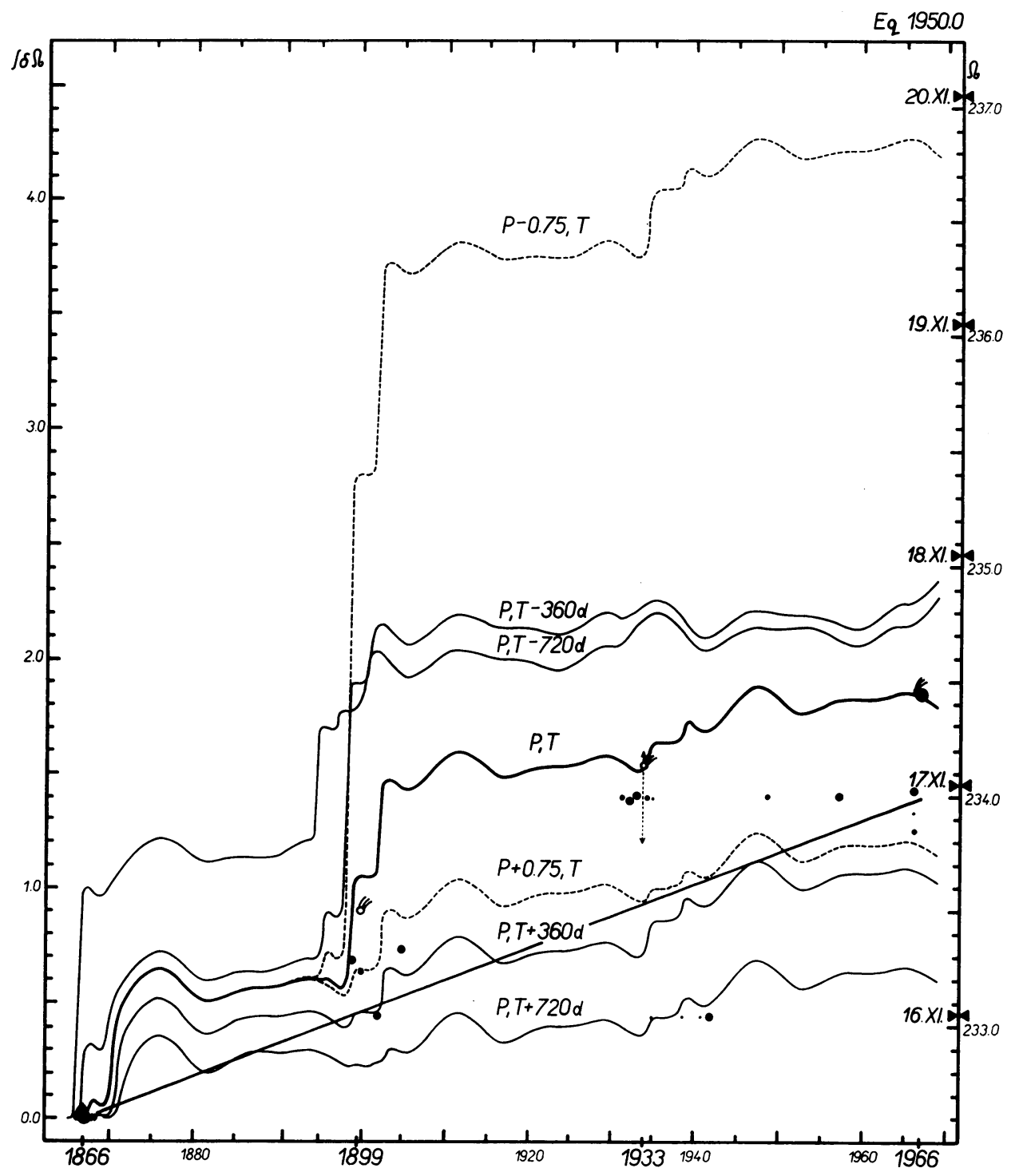

FIG. 1.

of short duration, e.g. 1 min. If several identical cameras, with same exposure time, same area covered, but of different apertures are used, not only will frequencies be obtained, but also the luminosity function can be determined. All-sky cameras would be convenient for recording the brightest meteors in the stream and for a determination of their frequency. Observations with instruments of the same type, 


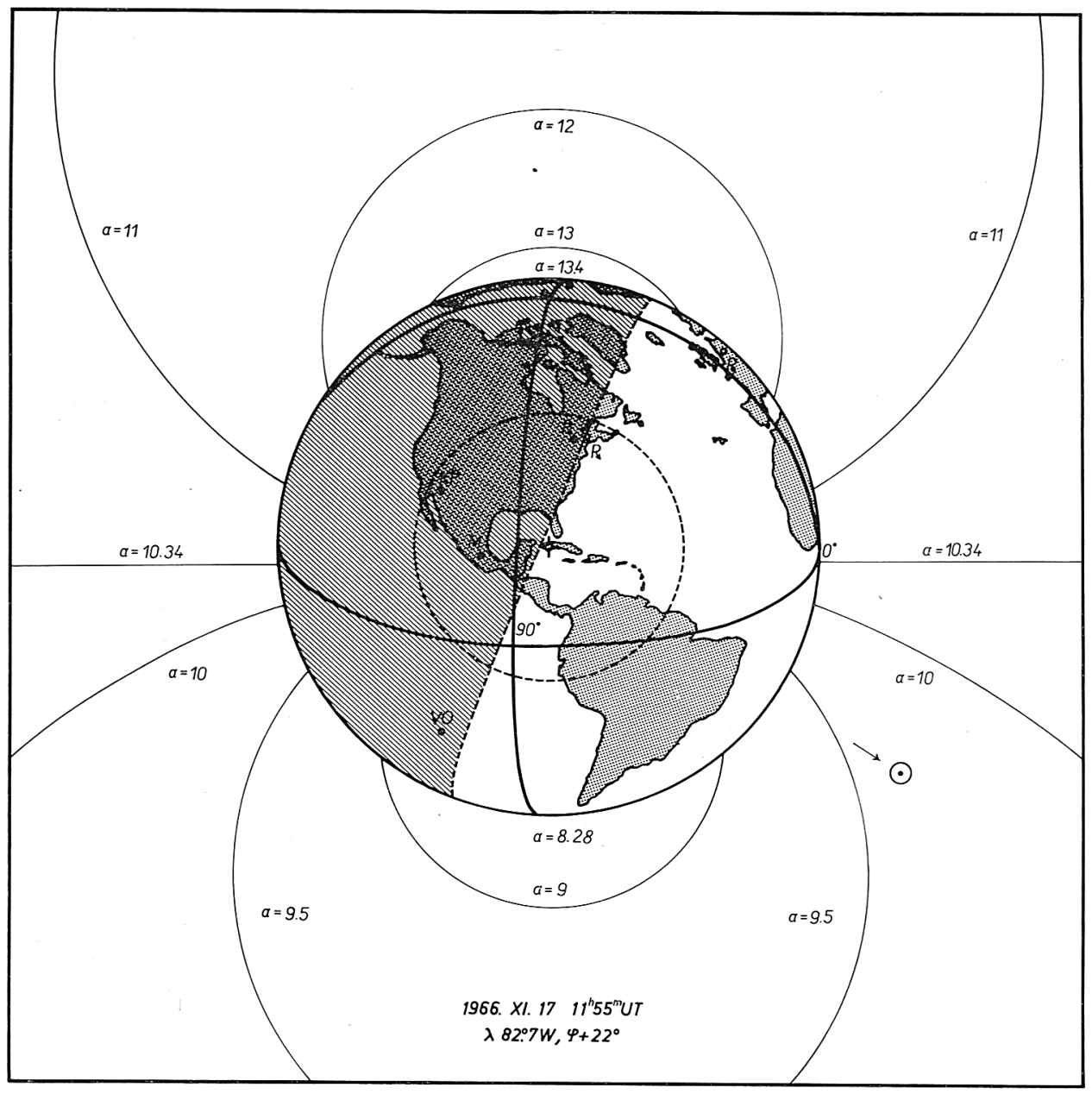

FIG. 2.

using plates or films of the same sensitivity but at different places, could determine the dependence of the frequency on the zeni thdistance of the radiant. Such standard methods of observation would lead to a better knowledge of the structure of meteor streams.

\section{Acknowledgements}

All calculations have been performed by Mrs. H. Ceplechová on the computer Minsk-22. To her I express my sincere thanks. 


\section{References}

Eckert, W.J., Brouwer, D., Clemence, G.M. (1951) Coordinates of the Five Outer Planets, Astr. Pap. Washington, 12.

Kazimirčak-Polonskaja, E.I., Beljaev, N.A., Astapovič, I.S., Terenteva, A.K. (1967) Astr. Zu., 44, 616.

Stoney, G.J., Downing, A. M.W. (1899) Proc. R. Soc. London, 64, 403.

\section{DISCUSSION}

Bronsten: I should like to note a good agreement of the curve of variations of the longitude of ascending node of the Leonid stream, obtained by Dr. Guth, with that calculated by KazimirčakPolonskaja et al. The dates and the ranges of two jumps in the longitude (1870 and 1898), caused by approaches with Saturn and Jupiter respectively, coincide on both curves.

Guth: The agreement of both values concerning the rate of the motion of node-line of Leonids is noteworthy as it has been obtained by two different methods: one by Cowell's, the other by the variation-of-elements method. 